\title{
Cooperative Aircraft Defense from an Attacking Missile
}

\author{
Eloy Garcia, David W. Casbeer, Khanh Pham, and Meir Pachter
}

\begin{abstract}
This paper addresses a three-body pursuit-evasion scenario where an Attacker missile using Pure Pursuit guidance pursues a Target aircraft and a Defender missile launched by a wingman aims at intercepting the Attacker before it reaches the aircraft. An optimal control problem is posed which captures the goal of the Target-Defender team, namely, to maximize the separation between Target and Attacker at the instant of capture of the Attacker by the Defender. The optimal control law provides the heading angles for the Target and the Defender team.
\end{abstract}

\section{INTRODUCTION}

Multi-agent pursuit-evasion scenarios represent important and challenging types of problems in control, aerospace, and robotics. In these types of problems one or more agents, called pursuers, try to maneuver and reach a relatively small distance with respect to one or more evaders, which move in order to escape the pursuers. Several types of pursuitevasion problems involving many agents have been recently studied. This problem is usually posed as a dynamic game [1], [2], [3]. Approaches based on dynamic Voronoi diagrams have been used in problems with several pursuers in order to capture an evader within a bounded environment as shown by Huang et.al. [2] and by Bakolas and Tsiotras [4]. On the other hand, [5] presented a receding-horizon approach that provides evasive maneuvers for a fixed-wing Unmanned Autonomous Vehicle (UAV) assuming a known model of the pursuer's input, state, and constraints. In [6], a multiagent scenario is presented where a number of pursuers are assigned to intercept a group of evaders where the dynamics and the goals of evaders are assumed to be known. In [7] a mobile sensor tries to track a target with a predefined trajectory while avoiding an attacker that tries to collide with and destroy the sensor. A Linear-Quadratic game is formulated between the sensor and the attacker where it is assumed that both have knowledge of the target's future trajectory.

Cooperation between two agents with the goal of evading a single pursuer has been addressed in [8]. Scott and Leonard [9] presented a scenario where two evaders search for coordinated strategies to evade a single pursuer but also to keep them close to each other.

E. Garcia is a contractor (Infoscitex Corp.) with the Control Science Center of Excellence, Air Force Research Laboratory, Wright-Patterson AFB, OH 45433. Corresponding Author: elgarcialinfoscitex.com

D. Casbeer is with the Control Science Center of Excellence, Air Force Research Laboratory, Wright-Patterson AFB, OH 45433.

K. Pham is with the Space Vehicles Directorate, Air Force Research Laboratory, Kirtland AFB, NM 87117.

M. Pachter is with the Department of Electrical Engineering, Air Force Institute of Technology, Wright-Patterson AFB, OH 45433.
Application of optimal pursuit strategies in autonomous vehicles heavily relies on the sensing capabilities of the pursuer in order for him to obtain accurate measurements about the evader; similarly, also the evader needs to track the pursuer in order to implement evasion strategies. The lack of accurate and timely information about the opposing team seriously complicates this problem. In the absence of sensing capabilities by the pursuer, the work in [10] presents an approach where the pursuer is aided by Unattended Ground Sensors (UGS) that provide limited and delayed information about the evader.

The present paper describes a three-agent pursuit-evasion problem where each agent has a defined role. The two-agent team consists of a Target and a Defender who cooperate; the Attacker agent is the opposition. The goal of the Attacker is to capture the Target while the Target tries to evade the Attacker and avoid capture. The Target cooperates with the Defender which pursues and tries to capture the Attacker before the latter captures the Target, see Fig. 1. Cooperation between the Target and the Defender is such that the Defender will capture the Attacker before the latter reaches the Target.

This scenario has been analyzed in the context of cooperative missile operations [11], [12]. Sensing capabilities of missiles and aircraft allow for implementation of complex pursuit and evasion strategies [13], [14]. Cooperative missile strategies have been recently studied by different researchers: for instance, [15] describes multi-missile cooperative attacks on a single stationary target (ship). Cooperation to control the impact time in order to simultaneously hit the ship is implemented as an additional loop to the typical Proportional Navigation (PN) guidance law. Similar work was presented in [16] and in [17] for moving targets.

Different types of cooperation have been recently proposed ([18], [19], [20], [21], [22], [23], [24]) for the TargetAttacker-Defender (TAD) scenario of Fig. 1. In these papers the Target represents an aircraft trying to survive a missile homing on it, the Attacker. The Defender represents another missile launched by the aircraft (or a wingman) in order to intercept and destroy the Attacker, to guarantee survival of the aircraft.

In [20] optimal policies (lateral acceleration for each agent including the Attacker) in numerical form are provided for the case of an aggressive Defender, that is, the Defender has a maneuverability advantage. A linear quadratic optimization problem is posed where the Defender's control effort weight is driven to zero to increase its aggressiveness. The work [21] provided a game theoretical analysis of the TAD problem using different guidance laws for both the Attacker and the 
Defender. The cooperative strategies shown in [22] allow for a maneuverability disadvantage for the Defender with respect of the Attacker and the results show that the optimal Target maneuver is either constant or arbitrary. Shaferman and Shima [23] implemented a multiple model adaptive estimator to identify the guidance law and parameters of the incoming missile and optimize a matched Defender strategy to minimize its control effort. In the recent paper [24] the authors analyze different types of cooperation assuming the Attacker is unaware of the Defender and its guidance law is known. Two different one-way cooperation strategies were discussed: when the Defender acts independently and the Target knows its future behavior cooperating with the Defender moves and vice versa. The two-way cooperation where both Target and Defender communicate continuously to exchange their states and controls is also addressed and it is shown to have a better performance that the other types of cooperation, as expected.

The work in the present paper provides jointly optimal solutions (heading angles of both the Target and the Defender) for a Pure Pursuit (PP) Attacker guidance law, based on the nonlinear dynamics of the three-agent engagement, as opposed to linearization approaches used in most of the papers discussed in the last paragraph. In addition, the Defender is not constrained to have similar or greater speed than the Attacker. The optimal cooperation between Target and Defender allows the latter to capture the Attacker even when it has a considerable speed disadvantage. Our solution shows the benefit of cooperation between Target and Defender during the whole duration of the engagement. The optimal control problem is solved as a Two-Point Boundary Value Problem (TPBVP). Simulation examples illustrate the efficiency of the cooperative behavior. These examples also show that, in general, little effort in terms of lateral acceleration is needed by both the Target and Defender, provided the Defender is initially properly positioned.

The paper is organized as follows. Section II describes the engagement scenario. Section III presents optimal policies for the Target and the Defender. This section also addresses details related to the numerical solution of the TPBVP and describes an application involving several potential launchers of the Defender missile. Examples are given in Section IV and Section V concludes the paper.

\section{Problem Statement}

The Target-Attacker-Defender engagement is illustrated in Fig. 1. The speeds of the Target, Attacker, and Defender are $V_{T}, V_{A}$, and $V_{D}$, respectively, which are assumed to be constant. It is assumed that the Attacker implements a PP guidance law.

The dynamics of the three vehicles in an inertial frame are given by:

$$
\begin{array}{ll}
\dot{x}_{T}=V_{T} \cos (\lambda+\phi), & \dot{y}_{T}=V_{T} \sin (\lambda+\phi) \\
\dot{x}_{A}=V_{A} \cos \lambda, & \dot{y}_{A}=V_{A} \sin \lambda \\
\dot{x}_{D}=V_{D} \cos \hat{\psi}, & \dot{y}_{D}=V_{D} \sin \hat{\psi}
\end{array}
$$

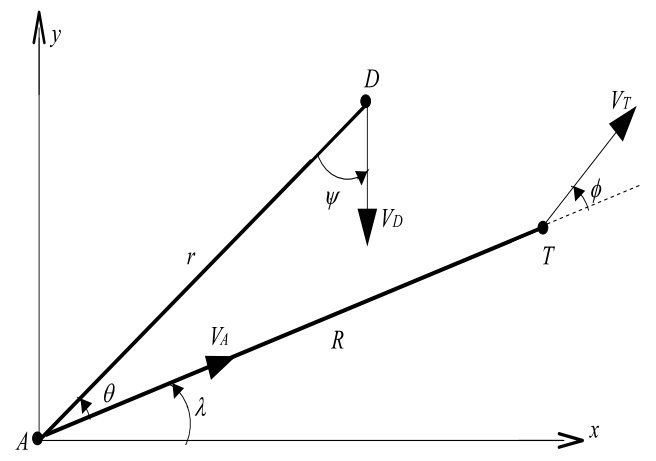

Fig. 1. Engagement geometry for the Target-Attacker-Defender problem where $\hat{\psi}=\psi+\theta+\lambda-\pi$.

Define the positive problem parameters $\alpha=V_{T} / V_{A}$ and $\beta=V_{D} / V_{A}$. In general, we have that the Attacker missile is faster than the Target aircraft, so that $\alpha<1$. In this work the speed of the Defender is not constrained to be equal or greater than the speed of the Attacker so $\beta$ is unrestricted in this sense; that is, $\beta$ could be equal to, less or greater than one. The variables $R$ and $r$ represent the separation between the Attacker and the Target and between the Attacker and the Defender, respectively. The positive constant $R_{c}$ represents the Attacker/Target capture radius and $r_{c}$ represents the Defender/Attacker capture radius.

The corresponding dynamics of the three-body engagement will be expressed using the reduced state space formed by the two separations $R$ and $r$ and by the angle between them, denoted by $\theta$. The objective is to find the optimal heading angles of the Target and the Defender in this relative frame which are denoted by $\phi$ and $\psi$, respectively, such that the distance $R$ is maximized while the separation $r$ becomes $r_{c}$ which represents capture of the Attacker by the Defender. Note that the relative heading angles can be easily transformed to heading angles with respect to the fixed coordinate axis $x$ using the angle $\lambda$. The use of the reduced state space provides a compact representation of the dynamics corresponding to this three-agent scenario.

\section{Cooperative Defense AGAinst An ATtACKer USING PP}

\section{A. Reduced order dynamical system}

To obtain a canonical form of the dynamics involved in the engagement shown in Fig. 1, we define the nondimensional variables using $R_{c}=1$ as follows: $R \rightarrow R / R_{c}$, $r \rightarrow r / R_{c}, r_{c} \rightarrow r_{c} / R_{c}, t \rightarrow t V_{A} / R_{c}$. Then, the dynamics corresponding to the variables of interest in this problem are given by

$$
\begin{aligned}
\dot{R} & =\alpha \cos \phi-1 \\
\dot{r} & =-\cos \theta-\beta \cos \psi \\
\dot{\theta} & =-\frac{\alpha}{R} \sin \phi-\frac{\beta}{r} \sin \psi+\frac{1}{r} \sin \theta
\end{aligned}
$$

with $R\left(t_{0}\right)=R_{0}, r\left(t_{0}\right)=r_{0}, \theta\left(t_{0}\right)=\theta_{0}$. Since $R$ is monotonically decreasing we can write an equivalent 
system of equations using a reduced number of variables and equations

$$
\begin{aligned}
\frac{d r}{d R} & =\frac{\cos \theta+\beta \cos \psi}{1-\alpha \cos \phi} \\
\frac{d \theta}{d R} & =\frac{1}{R r} \frac{\alpha r \sin \phi+\beta R \sin \psi-R \sin \theta}{1-\alpha \cos \phi}
\end{aligned}
$$

Further, since the terminal distance $R_{f}$ is free and $R_{f}>$ $R_{c}=1$, we let $\tau=R_{0}-R, 0 \leq \tau \leq \tau_{f}$ where $\tau_{f}=R_{0}-$ $R_{f}$. Similarly, $\tau_{f}$ is free and satisfies $\tau_{f} \leq R_{0}-R_{c}=R_{0}-1$.

We have that the state space is further reduced and is given by $\left[\begin{array}{ll}r & \theta\end{array}\right]^{T}$, and the dynamics can be expressed as follows:

$$
\begin{aligned}
& \frac{d r}{d \tau}=\frac{\cos \theta+\beta \cos \psi}{\alpha \cos \phi-1} \\
& \frac{d \theta}{d \tau}=\frac{\alpha r \sin \phi+\beta\left(R_{0}-\tau\right) \sin \psi-\left(R_{0}-\tau\right) \sin \theta}{r\left(R_{0}-\tau\right)(\alpha \cos \phi-1)}
\end{aligned}
$$

with

$$
\begin{aligned}
& r(0)=r_{0} \\
& \theta(0)=\theta_{0} .
\end{aligned}
$$

The objective of the Target-Defender team is to minimize $\tau_{f}$ while guaranteeing capture of the attacker as expressed by the terminal condition on $r$. The cost functional can be simply expressed by

$$
J=\int_{0}^{\tau_{f}} d \tau
$$

Then, the Hamiltonian is given by:

$$
\begin{aligned}
H= & 1+\frac{1}{\alpha \cos \phi-1}\left((\cos \theta+\beta \cos \psi) \lambda_{r}\right. \\
& +\frac{1}{r\left(R_{0}-\tau\right)}\left[\alpha r \sin \phi+\beta\left(R_{0}-\tau\right) \sin \psi\right. \\
& \left.\left.-\left(R_{0}-\tau\right) \sin \theta\right] \lambda_{\theta}\right)
\end{aligned}
$$

The co-state dynamics are found to be:

$$
\begin{aligned}
\frac{d \lambda_{r}}{d \tau} & =\frac{1}{r^{2}(\alpha \cos \phi-1)}(\beta \sin \psi-\sin \theta) \lambda_{\theta} \\
\frac{d \lambda_{\theta}}{d \tau} & =\frac{1}{\alpha \cos \phi-1}\left(\lambda_{r} \sin \theta+\frac{1}{r} \lambda_{\theta} \cos \theta\right)
\end{aligned}
$$

The terminal conditions for this free terminal time problem are as follows. The terminal state $r\left(\tau_{f}\right)$ is fixed and equal to $r_{c}$. The terminal state $\theta\left(\tau_{f}\right)$ is free, then we have $\lambda_{\theta}\left(\tau_{f}\right)=0$. The final terminal condition for optimality for this problem requires that $H\left(x^{*}\left(\tau_{f}\right), u^{*}\left(\tau_{f}\right), \lambda^{*}\left(\tau_{f}\right), \tau_{f}\right)=0$. In summary, the terminal conditions are:

$$
\begin{aligned}
r\left(\tau_{f}\right) & =r_{c} \\
\lambda_{\theta}\left(\tau_{f}\right) & =0 \\
1+\frac{1}{\alpha-1}\left(\cos \theta\left(\tau_{f}\right)+\beta\right) \lambda_{r}\left(\tau_{f}\right) & =0 .
\end{aligned}
$$

Theorem 1: Assume that the Attacker implements a PP guidance law to pursue the Target. Then the optimal control headings that maximize the separation between the Target and the Attacker and achieve $r\left(\tau_{f}\right)=r_{c}$ are given by

$$
\begin{aligned}
\sin \psi^{*} & =\frac{\lambda_{\theta}}{r \sqrt{\lambda_{r}^{2}+\lambda_{\theta}^{2} / r^{2}}} \\
\cos \psi^{*} & =\frac{\lambda_{r}}{\sqrt{\lambda_{r}^{2}+\lambda_{\theta}^{2} / r^{2}}}
\end{aligned}
$$

$$
\begin{aligned}
\sin \phi^{*} & =\frac{\left(-\alpha k_{1}\left(R_{0}-\tau\right)+k_{2}\right) \lambda_{\theta}}{\lambda_{\theta}^{2}+k_{1}^{2}\left(R_{0}-\tau\right)^{2}} \\
\cos \phi^{*} & =\frac{\alpha \lambda_{\theta}^{2}+k_{1} k_{2}\left(R_{0}-\tau\right)}{\lambda_{\theta}^{2}+k_{1}^{2}\left(R_{0}-\tau\right)^{2}}
\end{aligned}
$$

where

$$
\begin{aligned}
& k_{1}=\lambda_{r} \cos \theta-\frac{\lambda_{\theta}}{r} \sin \theta+\beta \sqrt{\lambda_{r}^{2}+\lambda_{\theta}^{2} / r^{2}} \\
& k_{2}=\sqrt{\lambda_{\theta}^{2}\left(1-\alpha^{2}\right)+k_{1}^{2}\left(R_{0}-\tau\right)^{2}} .
\end{aligned}
$$

Proof. In order to find the optimal heading angle equations involving $\psi^{*}$ we solve for this variable in the following:

$$
\frac{\partial H}{\partial \psi}=\frac{1}{\alpha \cos \phi-1}\left(\frac{\beta \lambda_{\theta}}{r} \cos \psi-\beta \lambda_{r} \sin \psi\right)=0
$$

which simplifies to

$$
\frac{\lambda_{\theta}}{r} \cos \psi=\lambda_{r} \sin \psi
$$

Using the relation $\cos ^{2} \psi=1-\sin ^{2} \psi$ we obtain the following

$$
\sin ^{2} \psi=\frac{\lambda_{\theta}^{2}}{r^{2}\left(\lambda_{r}^{2}+\lambda_{\theta}^{2} / r^{2}\right)}
$$

and we obtain (17). The expression (18) is found in a similar way by letting $\sin ^{2} \psi=1-\cos ^{2} \psi$ in (23).

In order to determine similar expressions for the optimal heading angle $\phi^{*}$ we solve for this variable in the following equation

$$
\begin{aligned}
\frac{\partial H}{\partial \phi}= & \frac{\alpha \lambda_{\theta} \cos \phi}{\left(R_{0}-\tau\right)(\alpha \cos \phi-1)} \\
& +\frac{(\cos \theta+\beta \cos \psi) \alpha \lambda_{r} \sin \phi}{(\alpha \cos \phi-1)^{2}} \\
& +\frac{\left(\alpha r \sin \phi+\beta\left(R_{0}-\tau\right) \sin \psi-\left(R_{0}-\tau\right) \sin \theta\right) \alpha \lambda_{\theta} \sin \phi}{r\left(R_{0}-\tau\right)(\alpha \cos \phi-1)^{2}} \\
= & 0
\end{aligned}
$$

which can be simplified to obtain

$$
k_{1}\left(R_{0}-\tau\right) \sin \phi=\lambda_{\theta} \cos \phi-\alpha \lambda_{\theta}
$$

and expressions (19) and (20) can be obtained using the relation $\sin ^{2} \phi+\cos ^{2} \phi=1$.

\section{B. Alternative solution using original dynamical system}

This problem can also be solved using the original system of equations (4)-(6) with

$$
\begin{aligned}
& R\left(t_{0}\right)=R_{0} \\
& r\left(t_{0}\right)=r_{0} \\
& \theta\left(t_{0}\right)=\theta_{0} .
\end{aligned}
$$

In this case the equivalent cost to (12) is to maximize the separation $R\left(t_{f}\right)$ which can be expressed as

$$
J=\int_{t_{0}}^{t_{f}} \dot{R} d t
$$

The Hamiltonian is given by

$$
\begin{aligned}
H= & 1-\alpha \cos \phi+(\alpha \cos \phi-1) \lambda_{R} \\
& -(\cos \theta+\beta \cos \psi) \lambda_{r} \\
& +\left(\frac{1}{r} \sin \theta-\frac{\alpha}{R} \sin \phi-\frac{\beta}{r} \sin \psi\right) \lambda_{\theta}
\end{aligned}
$$


and the co-state dynamics can be expressed as follows

$$
\begin{aligned}
& \dot{\lambda_{R}}=-\frac{\alpha \lambda_{\theta}}{R^{2}} \sin \phi \\
& \dot{\lambda_{r}}=\frac{\lambda_{\theta}}{r^{2}}(\sin \theta-\beta \sin \psi) \\
& \dot{\lambda_{\theta}}=-\lambda_{r} \sin \theta-\frac{\lambda_{\theta}}{r} \cos \theta
\end{aligned}
$$

The terminal conditions for this case are as follows.

$$
\begin{aligned}
r\left(t_{f}\right) & =r_{c} \\
\lambda_{R}\left(t_{f}\right) & =0 \\
\lambda_{\theta}\left(t_{f}\right) & =0 \\
1-\alpha-\lambda_{r}\left(t_{f}\right)\left(\cos \theta\left(t_{f}\right)+\beta\right) & =0
\end{aligned}
$$

Corollary 1: Assume that the Attacker implements a PP guidance law to pursue the Target. Then the optimal control headings that maximize the separation between the Target and the Attacker and achieve $r\left(t_{f}\right)=r_{c}$ are given by (17)(18) and by

$$
\begin{aligned}
\sin \phi^{*} & =\frac{\lambda_{\theta}}{R \sqrt{\left(1-\lambda_{R}\right)^{2}+\lambda_{\theta}^{2} / R^{2}}} \\
\cos \phi^{*} & =\frac{1-\lambda_{R}}{\sqrt{\left(1-\lambda_{R}\right)^{2}+\lambda_{\theta}^{2} / R^{2}}} .
\end{aligned}
$$

Proof. The proof follows similar steps than the proof of Theorem 1.

Remark. The disadvantage of using the original dynamics to solve the problem is that the a TPBVP needs to be solved with two extra variables and equations. The advantage is that a more compact expression for the control $\phi$ is obtained as seen in (31)-(32).

\section{Numerical solution}

The optimal headings derived in Theorem 1 and Corollary 1 are not useful in and of themselves due to the nonlinearities in the states, the co-states, and the control inputs. In practice, the derived optimal headings are used to numerically solve the TPBVP for the states and co-states. For the reduced order system in Section III-A, the numerical solution is found by substituting the optimal control headings in Theorem 1 into expressions (9), (10), (14), (15) with boundary conditions given by (11) and (16). In the second version, we substitute (17), (18), (31), and (32) into the dynamics (4)-(6) and (27)(29) with boundary conditions (24) and (30).

Typical TPBVP solvers require an initial guess of the solution. Solutions for $\theta_{0}=0$ are linear and their analytical expressions are given by (for the version shown in Section III-B)

$$
\begin{array}{ll}
R^{*}(t)=(\alpha-1) t+R_{0}, & \lambda_{R}^{*}(t)=0 \\
r^{*}(t)=-(1+\beta) t+r_{0}, & \lambda_{r}^{*}(t)=\frac{1-\alpha}{1+\beta} \\
\theta^{*}(t)=0, & \lambda_{\theta}^{*}(t)=0
\end{array}
$$

which can be used as an initial guess for problems with $\theta_{0} \neq 0$. For problems with $\theta_{0}$ far from zero, the solution can be obtained after a few iterations of the solver starting with a solution for $\theta_{0} \approx 0$ and incrementing $\theta_{0}$ to reach the required initial condition on $\theta$. The obtained solution at each iteration is used as an initial guess for the following iteration.

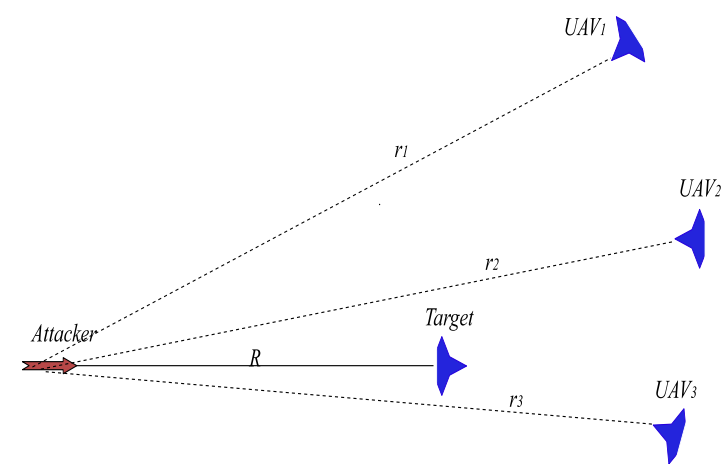

Fig. 2. Multiple friendly UAVs potentially helping the Target by launching the Defender against the Attacker

An additional issue in this problem is that the terminal value $\tau_{f}$ (respectively, $t_{f}$ ) is unknown. One approach to numerically find $\tau_{f}$ (respectively, $t_{f}$ ) is to relax one of the boundary conditions by introducing an unknown parameter and use Newton's method to update the initial guess for $\tau_{f}$ (respectively, $t_{f}$ ) and drive the unknown parameter to zero. Let $F$ represent either $\tau_{f}$ or $t_{f}$. For this particular problem it has been found that faster results can be obtained by relaxing the initial condition $r_{0}$. For this choice, we want $r_{0}\left(F_{k}\right)-r_{0} \rightarrow 0$, where $r_{0}\left(F_{k}\right)$ represents the relaxed initial condition when we provide $F_{k}$ as the guess for the final time and the index $k$ denotes iterations of Newton's method to update $F$. The equations corresponding to one of these iterations are

$$
\begin{aligned}
& F_{k}^{\prime}=F_{k}^{\prime}-\frac{r_{0}\left(F_{k}\right)-r_{0}}{\dot{r}_{0}\left(F_{k}\right)} \\
& F_{k}=F_{0}-F_{k}^{\prime}
\end{aligned}
$$

where the initial values of these iterations, $F_{0}, r_{0}\left(F_{0}\right)$, $\dot{r}_{0}\left(F_{0}\right)$, can be obtained, for instance, from the analytical solution (33).

\section{Application to multi-UAV}

In this section, we describe a multi-UAV scenario to illustrate how the solution to the cooperative defense problem presented in Section III-A or Section III-B can be used when there are different potential launchers of the Defender missile. Consider a battle scenario where a UAV is being pursued by an enemy missile and there are friendly UAVs in good positions to launch the Defender missile and help the distressed UAV to survive the attack, see Fig. 2. Since the targeted UAV is already traveling in direction to evade the Attacker, launching its own Defender would require an additional constraint on either the launched Defender or on the target UAV itself to turn back before firing. This maneuver could be problematic in practice. The other friendly UAVs may be aligned in such a way to have a better shot at the Attacker even if they are significantly farther from the missile than the Target. In addition, the other UAVs may be able to obtain better measurements of the Attacker missile due to their particular headings. 

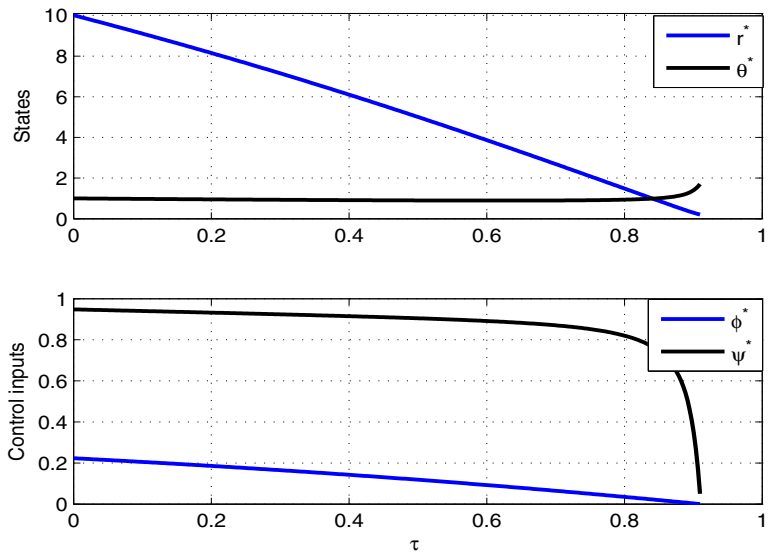

Fig. 3. Solution of Example 1 using the reduced order dynamical system
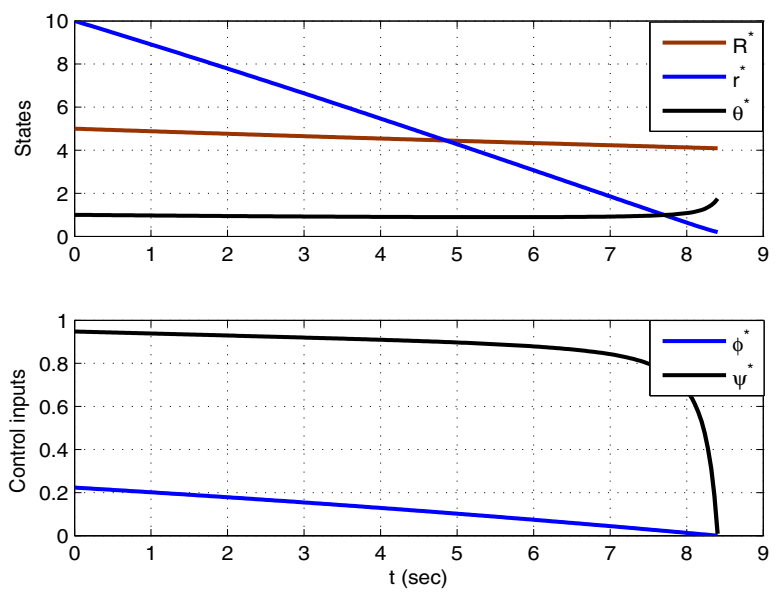

Fig. 4. Solution of Example 1 using the original dynamical system

Assuming that there exist a subset of $N$ friendly UAVs with acceptable headings labeled by $i=1, \ldots, N$ and that those UAVs know the current location of the Attacker and the Target then each one is able to solve the cooperative defense problem in a decentralized way to obtain optimal candidate policies $\left\{\psi_{i}^{*}, \phi_{i}^{*}\right\}$ and an associated optimal reward $R_{f_{i}}^{*}=$ $R_{i}^{*}\left(t_{f}\right)=R_{0}-\tau_{f_{i}}$. Then, each UAV only needs to transmit $R_{f_{i}}^{*}$ and $\phi_{i}^{*}$ to the Target which selects the overall best policy $\phi^{*}=\phi_{M}^{*},\left(M=\arg \max _{i}\left\{R_{f_{i}}^{*}\right\}\right)$ based on the maximum reward $R_{f}^{*}=\max _{i}\left\{R_{f_{i}}^{*}\right\}$. The Target only needs to respond to the selected launcher and start implementing the winning control input $\phi^{*}$. The selection of the maximum terminal separation $R\left(t_{f}\right)$ is highly desirable to safeguard the Target. The Target will have a better shot at evading and decide if additional countermeasures (such as releasing chaff and flares) are needed after the encounter of the Defender and the Attacker, if a higher separation from the Attacker is provided. This multi-UAV scenario represents a very interesting problem where the constraints on the initial heading angles of all possible UAVs that can fire a Defender missile, including the Target, deserves further attention. This problem represents a topic of future research.

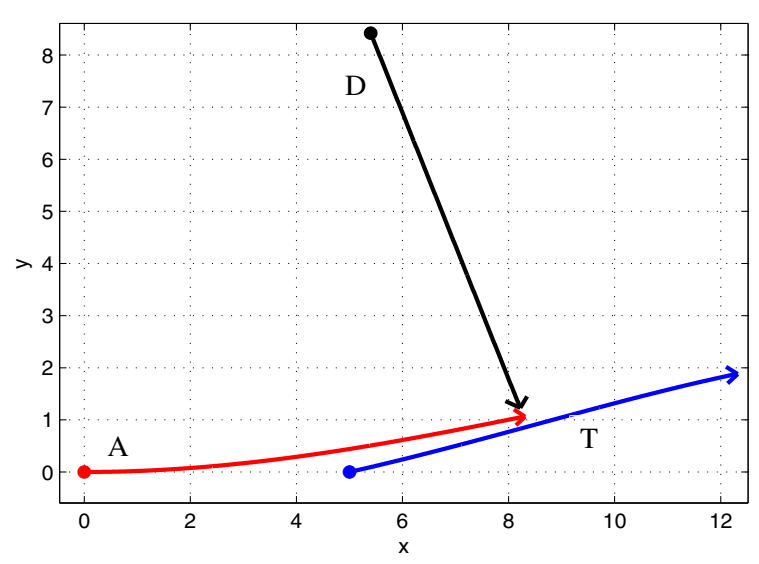

Fig. 5. Optimal trajectories for Example 1 in a fixed Cartesian frame of Target $\longrightarrow$, Attacker $\longrightarrow$, and Defender $\longrightarrow$

\section{EXAMPLES}

Example 1: Comparison of solutions using reduced and original dynamical systems. The initial conditions are given by $R_{0}=5, r_{0}=10$, and $\theta_{0}=1 \mathrm{rad}$. We also have that $V_{A}=$ $1, R_{c}=1$, and $r_{c}=0.2$. The relative velocity parameters are given by $\alpha=0.9$ and $\beta=0.92$. Note that the value of $\beta$ indicates that the Defender is slower than the Attacker. Fig. 3 shows the optimal solutions $r^{*}(\tau)$ and $\theta^{*}(\tau)$ using the reduced dynamical system version described in Section IIIA. The bottom of the same figure shows the optimal headings $\phi^{*}(\tau)$ and $\psi^{*}(\tau)$. The terminal value of $\tau$ is approximately $\tau_{f}=0.91$. Note that information about the separation $R$ is embedded in the axis $\tau$, for instance the terminal separation in this example is $R_{f}=R_{0}-\tau_{f}=4.09$.

Fig. 4 shows the solution using the approach shown in Section III-B. It can be seen that the same solutions are obtained as expected and the corresponding terminal time is approximately $t_{f}=8.405 \mathrm{sec}$. It can also be seen that the terminal value $R_{f}=4.09$ is equivalent to the one found using the terminal value of $\tau_{f}$. Fig. 5 shows the trajectories of the three agents in a fixed coordinate frame.

Remark. Results from simulations show that optimal trajectories require, in general, little effort by the Defender and the Target in terms of lateral accelerations. Note that the angles $\phi^{*}$ and $\psi^{*}$ are relative angles with respect to the relative geometry formed by the separations $R$ and $r$ and the angle between them $\theta$. For instance, although the angle $\psi^{*}$ changes rapidly by the end of the engagement the Defender does not implement this heading but $\hat{\psi}^{*}$. This change in $\psi^{*}$ is compensated by the change in $\theta^{*}$ during the similar time interval and what we have is that the Defender actual heading, $\hat{\psi}^{*}$, does not change rapidly as shown by the optimal Defender trajectory in Fig. 5.

Remark. For given initial conditions and given parameter $\alpha$, it is necessary to solve the TPBVP to determine if the Defender missile is fast enough to intercept the Attacker before the latter captures the Target. If, by solving the optimal control problem, we find that $R\left(t_{F}\right)<R_{c}$ then, for those initial conditions the Defender is unable to help the 


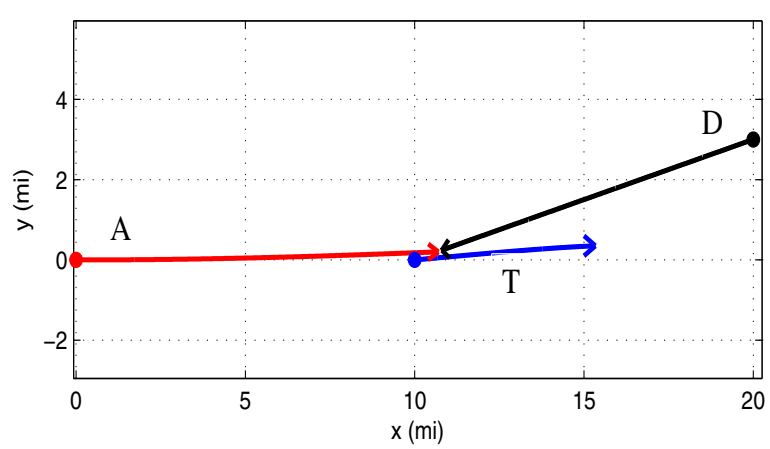

Fig. 6. Optimal trajectories for Example 2 in a fixed Cartesian frame of Target $\longrightarrow$, Attacker $\longrightarrow$, and Defender $\longrightarrow$

Target to escape since the optimal strategy does not provide a large enough final separation $R\left(t_{f}\right)$.

Example 2: Multi-UAV. Consider a scenario similar to the one shown in Fig. 2 where the initial separation between Target and Attacker is $R_{0}=10$ miles. The Target's initial position is at $(10,0)$ and heading away from the Attacker. Three friendly UAVs $i=1,2,3$ are within range and with an acceptable heading to potentially fire the Defender missile. The UAVs initial positions are $(18,7),(20,3)$, and $(21,-1)$; the position coordinates of all agents are measured in miles with respect to a fixed Cartesian frame with origin at the Attacker initial position. The speeds of the Target is $V_{T}=$ $0.25 \mathrm{mi} / \mathrm{sec}$ and the speed of the Attacker is $V_{A}=0.5 \mathrm{mi} / \mathrm{sec}$, that is, $\alpha=0.5$. Each UAV can launch a Defender missile with speed $V_{D_{i}}=0.45 \mathrm{mi} / \mathrm{sec}$, for $i=1,2,3$, and we have that $\beta_{i}=0.9$, for $i=1,2,3$. Each UAV computes its optimal solution to the cooperative defense problem and the potential final separations are given by $R_{f_{1}}=4.62, R_{f_{2}}=4.67$, and $R_{f_{3}}=4.51$. The selected launcher is UAV 2 and the the optimal trajectories for the selected launched Defender are shown in Fig. 6.

\section{CONCLUSion}

We presented a practical cooperative control problem that considers a missile homing into an aircraft, and the aircraft is aided by an intercepting missile. Cooperation between the aircraft and the friendly missile was described and an optimal control problem was solved which returns the optimal heading angles for these two agents. This problem was solved when the attacking missile uses a PP guidance law. The solutions presented in this paper do not restrict the Defender to have similar or greater speed than the Attacker. This means that interception of an attacking missile can be accomplished using a slower missile by implementing an appropriate cooperative strategy between the Target and the Defender. Simulation examples show that, in general, little effort in terms of lateral acceleration is needed by both the Target and Defender when the Defender is properly positioned at the initial time of the engagement. Explicit consideration of Target and Defender dynamic constraints and other Attacker guidance laws will be studied in future research.

\section{REFERENCES}

[1] S. A. Ganebny, S. S. Kumkov, S. Le Ménec, and V. S. Patsko, "Model problem in a line with two pursuers and one evader," Dynamic Games and Applications, vol. 2, no. 2, pp. 228-257, 2012.

[2] H. Huang, W. Zhang, J. Ding, D. M. Stipanovic, and C. J. Tomlin, "Guaranteed decentralized pursuit-evasion in the plane with multiple pursuers," in 50th IEEE Conference on Decision and Control and European Control Conference, 2011, pp. 4835-4840.

[3] K. Pham, "Risk-averse based paradigms for uncertainty forecast and management in differential games of persistent disruptions and denials," in American Control Conference, 2010, pp. 842-849.

[4] E. Bakolas and P. Tsiotras, "Optimal pursuit of moving targets using dynamic voronoi diagrams," in 49th IEEE Conference on Decision and Control, 2010, pp. 7431-7436.

[5] J. Sprinkle, J. M. Eklund, H. J. Kim, and S. Sastry, "Encoding aerial pursuit/evasion games with fixed wing aircraft into a nonlinear model predictive tracking controller," in 43rd IEEE Conference on Decision and Control, 2004, pp. 2609-2614.

[6] M. G. Earl and R. DAndrea, "A decomposition approach to multivehicle cooperative control," Robotics and Autonomous Systems, vol. 55, no. 4, pp. 276-291, 2007.

[7] D. Li, G. Chen, E. Blasch, and K. Pham, "Sensor attack avoidance: linear quadratic game approach," in 12th International Conference on Information Fusion, 2009, pp. 1131-1138.

[8] Z. E. Fuchs, P. P. Khargonekar, and J. Evers, "Cooperative defense within a single-pursuer, two-evader pursuit evasion differential game," in 49th IEEE Conference on Decision and Control, 2010, pp. 30913097.

[9] W. Scott and N. E. Leonard, "Pursuit, herding and evasion: A threeagent model of caribou predation," in American Control Conference, 2013, pp. 2978-2983.

[10] K. Krishnamoorthy, D. Casbeer, P. Chandler, M. Pachter, and S. Darbha, "UAV search and capture of a moving ground target under delayed information," in 51st IEEE Conference on Decision and Control, 2012, pp. 3092-3097.

[11] R. L. Boyell, "Defending a moving target against missile or torpedo attack," IEEE Transactions on Aerospace and Electronic Systems, vol. AES-12, no. 4, pp. 522-526, 1976.

[12] _ _ "Counterweapon aiming for defence of a moving target," IEEE Transactions on Aerospace and Electronic Systems, vol. AES-16, no. 3, pp. 402-408, 1980.

[13] P. Zarchan, Tactical and strategic missile guidance. AIAA Progress in Aeronautics and Astronautics, Reston, VA, 1997, vol. 176.

[14] G. Siouris, Missile guidance and control systems. New York, Springer, 2004.

[15] I.-S. Jeon, J.-I. Lee, and M.-J. Tahk, "Impact-time-control guidance law for anti-ship missiles," IEEE Transactions on Control Systems Technology, vol. 14, no. 2, pp. 260-266, 2006.

[16] J.-I. Lee, I.-S. Jeon, and M.-J. Tahk, "Guidance law to control impact time and angle," IEEE Transactions on Aerospace and Electronic Systems, vol. 43, no. 1, pp. 301-310, 2007.

[17] V. Shaferman and T. Shima, "Cooperative optimal guidance laws for imposing a relative intercept angle," in AIAA Guidance, Navigation, and Control Conference, 2012.

[18] A. Perelman, T. Shima, and I. Rusnak, "Cooperative differential games strategies for active aircraft protection from a homing missile," Journal of Guidance, Control, and Dynamics, vol. 34, no. 3, pp. 761-773, 2011.

[19] I. Rusnak, "The lady, the bandits, and the bodyguards-a two team dynamic game," in 16th IFAC World Congress, 2005.

[20] I. Rusnak, H. Weiss, and G. Hexner, "Guidance laws in target-missiledefender scenario with an aggressive defender," in 18th IFAC World Congress, vol. 18, no. Pt 1, 2011, pp. 9349-9354.

[21] A. Ratnoo and T. Shima, "Guidance strategies against defended aerial targets," Journal of Guidance, Control, and Dynamics, vol. 35, no. 4, pp. 1059-1068, 2012.

[22] T. Shima, "Optimal cooperative pursuit and evasion strategies against a homing missile," Journal of Guidance, Control, and Dynamics, vol. 34, no. 2, pp. 414-425, 2011.

[23] V. Shaferman and T. Shima, "Cooperative multiple-model adaptive guidance for an aircraft defending missile," Journal of Guidance, Control, and Dynamics, vol. 33, no. 6, pp. 1801-1813, 2010.

[24] O. Prokopov and T. Shima, "Linear quadratic optimal cooperative strategies for active aircraft protection," Journal of Guidance, Control, and Dynamics, vol. 36, no. 3, pp. 753-764, 2013. 